Tema: Iniciação cientifico-tecnológica

\title{
ESTUDO DO COMPORTAMENTO TERMOMECÂNICO DO AÇO API 5L X80M - PSL2 MICROLIGADO AO Nb-Ti UTILIZANDO ENSAIOS ISOTÉRMICOS DE TORÇÃO A QUENTE*
}

\author{
Camila Simon Christ $^{1}$ \\ Luciana Nogueira ${ }^{1}$ \\ Marcelo Lucas Pereira Machado ${ }^{2}$ \\ Fernanda Huebra ${ }^{3}$ \\ José Brunoro 4
}

\section{Resumo}

O desenvolvimento de aços de alta resistência associados à boa tenacidade é uma alternativa para redução de custos na construção e instalação de oleodutos e gasodutos, devido à possibilidade de redução da espessura dos tubos sem que seu desempenho seja afetado. Os aços da classe API (American Petroleum Institute) pertencem ao grupo de aços ARBL (Alta Resistência Baixa Liga) que possuem as combinações desejáveis de resistência à tração, ductilidade, tenacidade e soldabilidade. No processo de laminação a quente o comportamento dos aços está diretamente relacionado aos parâmetros de processamento como temperatura, deformação, taxa de deformação, taxa de resfriamento e tempo entre passes. O controle desses parâmetros é importante na determinação da microestrutura e propriedades mecânicas do material. O objetivo deste trabalho é estudar o comportamento termomecânico do aço API 5L X80M-PSL2 através de ensaios de torção a quente isotérmicos. Através desses ensaios serão determinados a tensão e deformação de pico, tensão e deformação crítica e a porcentagem de amaciamento do material.

Palavras-chave: Ensaio de torção a quente; API 5L X80M - PSL2; Recristalização dinâmica.

\section{STUDY OF THE THERMOMECHANICAL BEHAVIOR OF API 5L X80M STEEL - PSL2 MICROALLOYED Nb-Ti USING ISOTHERMAL HOT TORSION}

\section{Abstract}

The development of high strength steels associated with good toughness is an alternative to reduce costs in the construction and installation of pipelines, due to the possibility of reducing the tubes thickness without its performance being affected. The API steels (American Petroleum Institute) belong to the class HSLA (High Strength Low Alloy) possessing the desirable combinations of tensile strength, ductility, toughness and weldability. In hot rolling process the steels behavior is directly related to the processing parameters such as temperature, strain, strain rate, cooling rate and interpass time. The control of these parameters is important to determine the microstructure and mechanical properties of the material. The main of this work is to study the thermomechanical behavior of API 5L PSL2 $\mathrm{X} 80 \mathrm{M}$ steel through isothermal hot torsion test. Through these tests will be determined the peak stress and strain, critical stress and strain and the softening percentage of the material. Keywords: Isothermal hot torsion; API 5L X80M - PSL2; Dynamic recrystallization.

1 Bolsista de Iniciação Científica, Estudante do Curso de Eng. Metalúrgica, IFES, Vitória, ES, Brasil. 2 Professor Doutor e Titular, Depto. Eng. Metalúrgica e de Materiais, IFES, Vitória, ES, Brasil.

3 Engenheira Mecânica, Analista em Assistência Técnica a Clientes da ArcelorMittal Tubarão, Aluna de Mestrado em Engenharia Metalúrgica e de Materiais do IFES, Vitória, ES, Brasil.

4 Engenheiro Mecânico, Professor, Coordenadoria do Curso Técnico em Mecânica, Aluno do Programa de Pós-Graduação em Eng. Metalúrgica e de Materiais, IFES, Vitória, ES, Brasil.

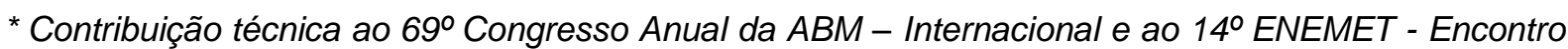
Nacional de Estudantes de Engenharia Metalúrgica, de Materiais e de Minas, 21 a 25 de julho de 2014, São Paulo, SP, Brasil.
} 


\section{INTRODUÇÃO}

Os principais processos industriais de conformação a quente são a laminação, a extrusão e o forjamento. Esses processos podem ser caracterizados em termos das variáveis relevantes à simulação por torção a quente sendo essas, em geral, a temperatura, a deformação, a taxa de deformação e o tempo entre deformações [1]. $O$ processo de conformação a quente consiste, inicialmente, em aquecer o material até a temperatura de encharque para a austenitização, realizar deformações programadas nas etapas de desbaste e de acabamento e em seguida promover 0 resfriamento controlado do material. A etapa de acabamento, durante o processo de laminação de tiras a quente é realizada com curtos tempos de espera entre passes. Sendo o intervalo entre passes pequenos, a recristalização estática poderá não se completar, podendo ter o acúmulo de deformação de um passe para o outro e, consequentemente, o processo passa a ser controlado pela recristalização dinâmica ou metadinâmica. Uma técnica experimental capaz de reproduzir estas condições de processamento e que permite investigar os mecanismos que estão operando é o ensaio de torção a quente, através das curvas de escoamento plástico e do acompanhamento microestrutural. Com ensaios de torção a quente pode-se realizar seqüências de passes impondo parâmetros de processamento tais como a temperatura de reaquecimento, a taxa de resfriamento, a quantidade de deformação, a taxa de deformação e o tempo de espera entre passes.

Pela analise da a evolução da curva de escoamento plástico em conjunto com observações microestruturais pode-se determinar o mecanismo de amaciamento dominante em cada uma das etapas de processamento, permitindo assim, determinar os eventos característico da laminação a quente e projetar seqüências de deformações que aperfeiçoam o processamento [2].

A laminação é um exemplo de trabalho mecânico a quente, sendo uma etapa de extrema importância, pois é através dela que se obtêm as formas adequadas dos produtos em aço para uso comercial (chapas, perfis, barras) [3].

\section{MATERIAIS E MÉTODOS}

\subsection{Material Utilizado}

O material utilizado foi um aço API $5 \mathrm{~L}$ X80 (com alto $\mathrm{Nb}$ ) produzido no Laminador de Tiras a Quente da ArcelorMittal Tubarão. A amostra foi fornecida pela TSA (Tubos Soldados do Atlântico), cuja a composições químicas estão apresentadas na Tabela 1 seguinte. A análise química foi realizada utilizando um espectrômetro Oxford Instruments, modelo Foundry-Master Pro do laboratório de redução do IFES.

Tabela 1 - Composição química do API $5 \mathrm{~L}$ X80 (com alto Nb)

\begin{tabular}{|c|c|c|c|c|c|c|c|c|c|c|}
\hline \multicolumn{10}{|c|}{ 'Elementos (\% em peso) } \\
\hline $\mathrm{C}$ & $\mathrm{Si}$ & $\mathrm{Mn}$ & $\mathrm{P}$ & $\mathrm{S}$ & $\mathrm{Al}$ & $\mathrm{N}$ & $\mathrm{Nb}$ & $\mathrm{Ti}$ & $\mathrm{Ca}$ & $\mathrm{Fe}$ \\
\hline$\leq 0,100$ & $\leq 0,300$ & $\leq 1,700$ & $\leq 0,018$ & $\leq 0,005$ & $\leq 0,050$ & $\leq 0,010$ & $\sim 0,090$ & $\leq 0,030$ & $\leq 0,005$ & Balanço \\
\hline
\end{tabular}

\subsection{Preparação dos Corpos de Prova}

Os Corpos de prova de torção foram usinados a partir de uma chapa de 15,88 $\mathrm{mm} x$ $1,500 \mathrm{~mm} \times 500 \mathrm{~mm}$, com diâmetro útil de $5 \mathrm{~mm}$ e comprimento útil de $20 \mathrm{~mm}$, como representado na Figura 1. O dimensionamento dos corpos de prova, diâmetro e

* Contribuição técnica ao 69 Congresso Anual da ABM - Internacional e ao 14ํㅡㄹ ENET - Encontro Nacional de Estudantes de Engenharia Metalúrgica, de Materiais e de Minas, 21 a 25 de julho de 2014, São Paulo, SP, Brasil. 


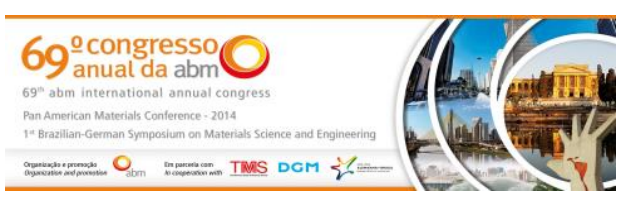

comprimento úteis, foram otimizados para atender à capacidade de máximo torque do equipamento de torção e, assim, obter maiores taxas de deformação.
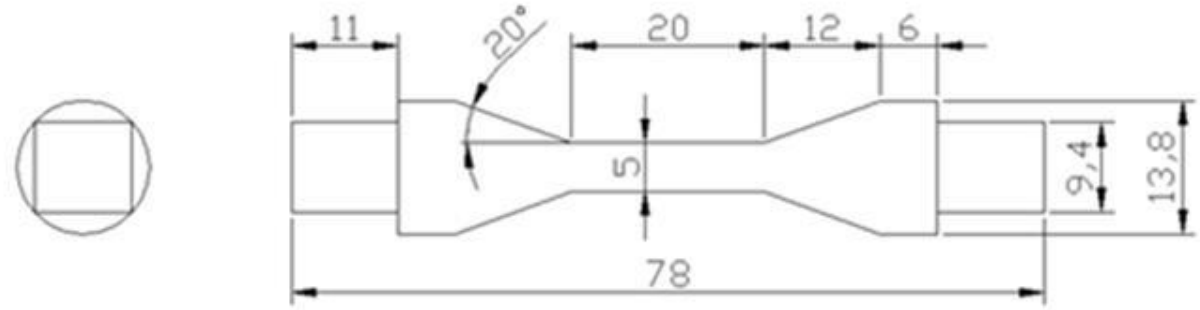

Figura 1 - Desenho esquemático do corpo de prova.

\subsection{Experimentos}

Todos os ensaios foram realizados utilizando-se uma máquina horizontal de torção a quente INSTRON, modelo 55MT do laboratório de conformação mecânica do IFES. Colocou-se um tubo de quartzo de aproximadamente $40 \mathrm{~mm}$ com injeção de argônio e gás $\mathrm{CO}_{2}$ para criar uma atmosfera inerte durante o ensaio, ocasionar o tratamento térmico de têmpera e evitar a corrosão do corpo de prova. Colocou-se uma bobina de cobre com forno de indução em volta do tubo de quartzo para que a temperatura no corpo de prova seja mais homogênea e o controle de temperatura foi feita através de um termopar do tipo $\mathrm{K}$ (Cromel-Alumel) inserido na extremidade do corpo de prova, minimizando quaisquer erros do ensaio.

\subsubsection{Ensaios isotérmicos e contínuos}

Os corpos de prova foram aquecidos até a temperatura de $1.200^{\circ} \mathrm{C}$ a uma taxa média de $2^{\circ} \mathrm{C} / \mathrm{s}$, e mantidos nesta temperatura por um período de 3 minutos, a seguir resfriados com uma taxa média de $1^{\circ} \mathrm{C} / \mathrm{s}$ até a temperatura de ensaio e mantidos nesta temperatura por 1 minuto para eliminação dos efeitos de gradientes térmicos antes do início da deformação.

As temperaturas utilizadas nos ensaios isotérmicos foram $1.150^{\circ} \mathrm{C}, 1.100^{\circ} \mathrm{C}$, $1.000^{\circ} \mathrm{C}, 950^{\circ} \mathrm{C}$ e $850^{\circ} \mathrm{C}$, as taxas de deformação foram de $0,2 \mathrm{~s}^{-1}$ e deformação máxima de 3. Para estudar o comportamento do material em relação a taxa de deformação foram realizados ensaios na temperatura de $1.150^{\circ} \mathrm{C}$ e taxas de deformação de $0,2^{-1} ; 0,4^{-1} ; 0,8^{-1}$ com deformação máxima de 3 . Na figura 2 é possível observar esquematicamente o ciclo termomecânico nos ensaios isotérmicos.

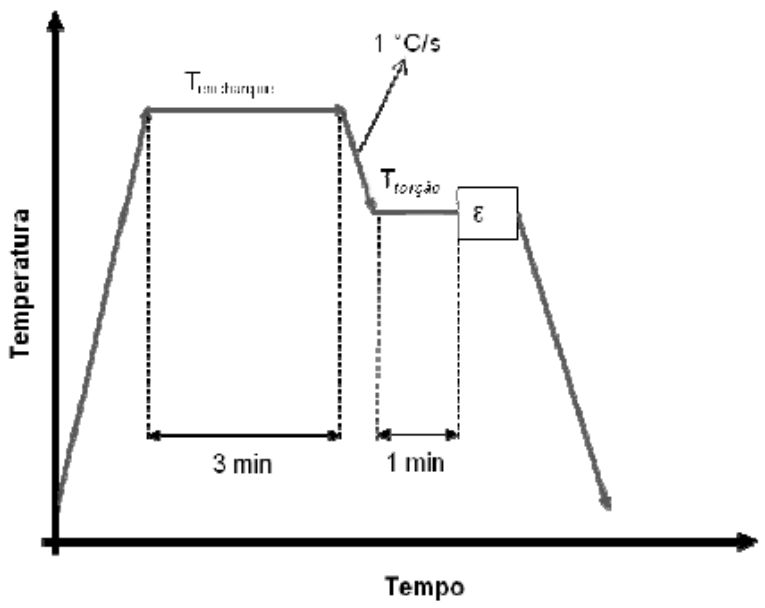

Figura 2: Representação esquemática do ciclo termomecânico utilizado nos ensaios isotérmicos.

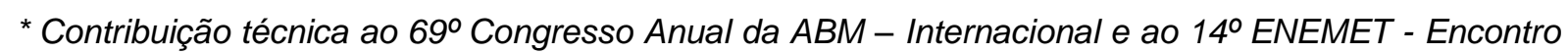
Nacional de Estudantes de Engenharia Metalúrgica, de Materiais e de Minas, 21 a 25 de julho de 2014, São Paulo, SP, Brasil. 


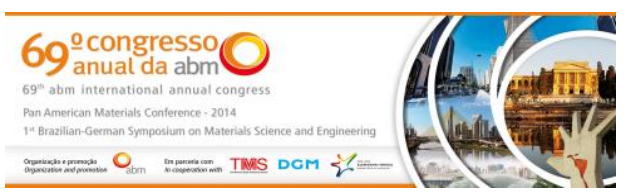

Após a deformação as amostras foram temperadas utilizando gás $\mathrm{CO}_{2}$, com 0 objetivo de congelar o tamanho do grão austenítico para análise microestrutural.

\section{RESULTADOS E DISCUSSÃO}

\subsection{Ensaios Isotérmicos Contínuos}

As condições de realização destes ensaios descritos no item 2.3.1. As curvas de escoamento plástico são representadas por ensaios com diferentes taxas de deformação e temperatura constante (Figuras 3 a 6) e por diferentes temperaturas e taxa de deformação constante (Figura 7).

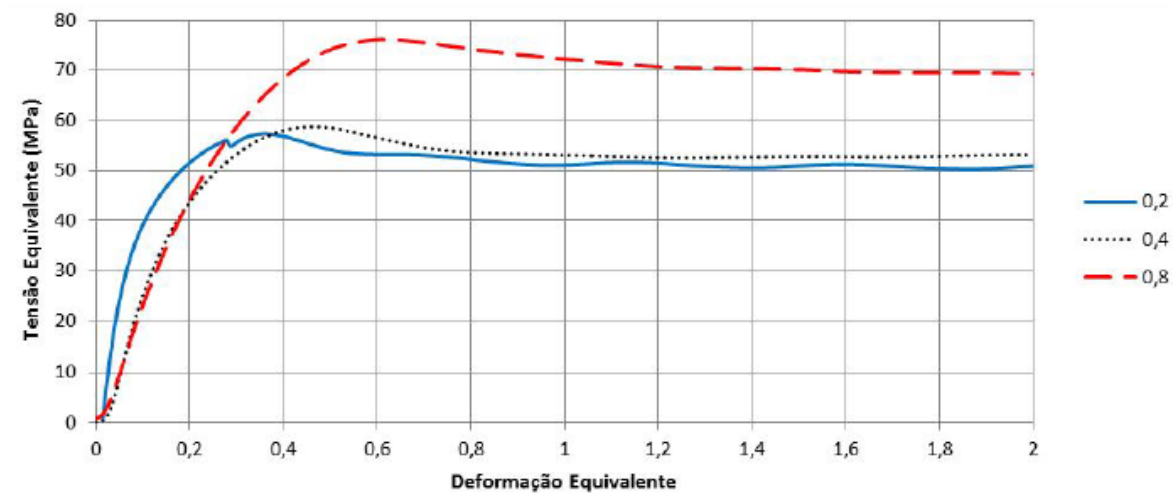

Figura 3: Gráfico dos ensaios isotérmicos $\left(1.150^{\circ} \mathrm{C}\right)$ com diferentes taxas de deformação para o aço API SL X80.

Observa-se na Figura 3 que as curvas de tensão equivalente em função da deformação equivalente para uma temperatura de $1.150^{\circ} \mathrm{C}$ apresentam um comportamento típico de materiais que se recristalizam dinamicamente, ou seja, a tensão aumenta com a deformação até atingir um pico caindo logo a seguir até um estado estacionário. Quanto maior for a taxa de deformação maior será a tensão de pico e também há um deslocamento nas curvas para a direita. Este comportamento pode ser observado em estudos realizados para outros aços através de ensaios de torção [4-6].

Para melhor compreensão desse comportamento do aço quando submetido às diferentes taxas de deformação, podemos verificar a tensão em função do tempo de duração dos testes na figura 4. Assim, podemos observar o comportamento da tensão como se os testes fossem realizados simultaneamente.

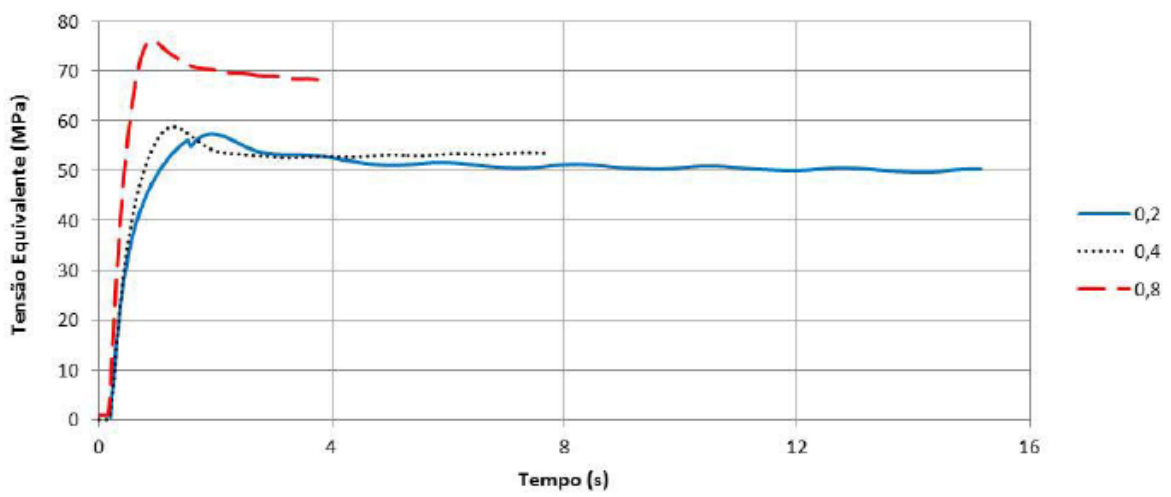

Figura 4: Curvas Tensão equivalente versus tempo de deformação de deformação obtidas por ensaios de torção a quente para um aço API SL X80 a diferentes taxas de deformação a temperatura de $1.150^{\circ} \mathrm{C}$.

* Contribuição técnica ao 69 Congresso Anual da ABM - Internacional e ao 14ํㅡㄹ ENET - Encontro Nacional de Estudantes de Engenharia Metalúrgica, de Materiais e de Minas, 21 a 25 de julho de 2014, São Paulo, SP, Brasil. 


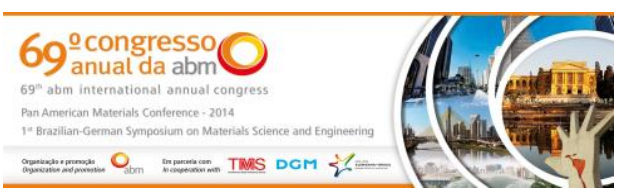

Observa-se que, para uma mesma deformação, quanto maior a taxa de deformação utilizada, menor o tempo o tempo gasto para se realizar o ensaio. Isso devido a elevada geração de discordâncias em um menor tempo, tendo uma tensão critica em menor tempo.

Na figura 5 são mostradas as curvas da taxa de encruamento $(\theta)$ versus tensão equivalente $(\sigma)$ para ensaios realizados a diferentes taxas de deformação e temperatura de $1.150^{\circ} \mathrm{C}$.

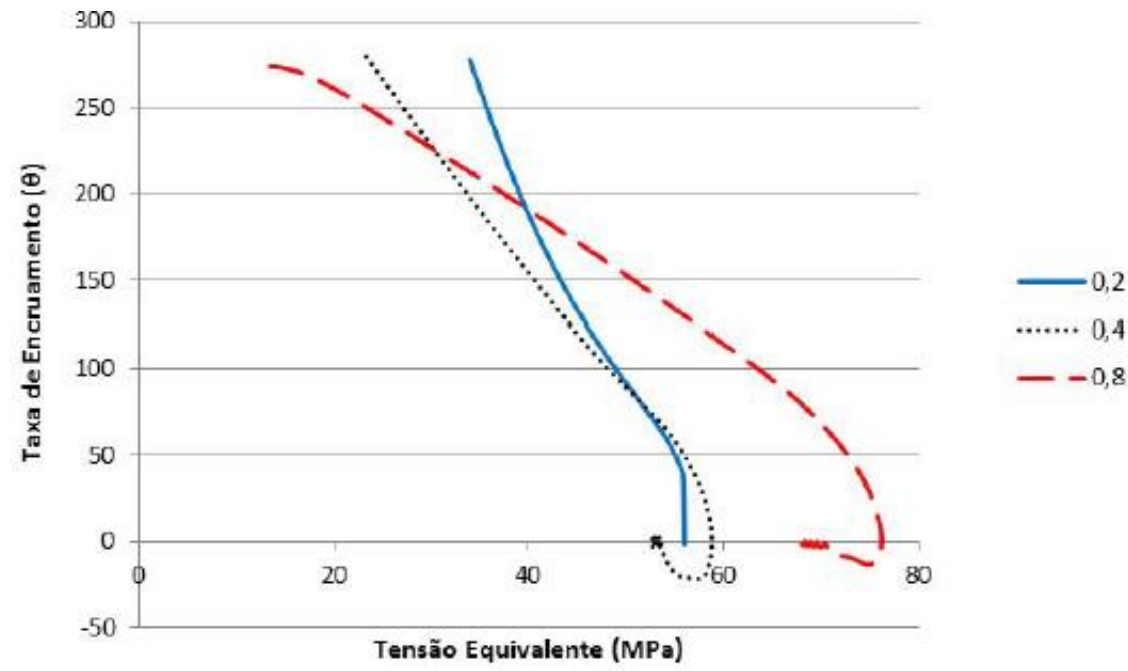

Figura 5: Variação da Taxa de Encruamento $(\theta)$ versus a tensão equivalente para um aço API SL X80 a diferentes temperaturas, taxa de deformação e temperatura de $1.150{ }^{\circ} \mathrm{C}$.

Assim, pela figura 5, observa-se que para taxa de deformação maior $\left(0,8 \mathrm{~s}^{-1}\right)$ não foi observado o ponto de inflexão, ou seja, não é possível indicar o valor da deformação critica. O que também foi observado em outro estudo com um aço SAE 1518 [7]. Isso ocorre por causa da elevada taxa de deformação, que tende a gerar mais discordâncias em um menor espaço de tempo, dificultando a visualização do ponto de inflexão. Sendo que a recristalização fica melhor visualizada em taxas de deformações menores.

Para melhor vizualização do ponto de inflexão, deriva-se as curvas da figura 5, obtem-se a tensão critica $\left(\sigma_{c}\right)$ através do ponto máximo das curvas, sendo que para a taxa de deformação de $0,8 \mathrm{~s}^{-1}$ é mais difícil identificar a tensão critica $\left(\sigma_{c}\right)$, por não haver a presença de pico. Como mostra a figura 6.

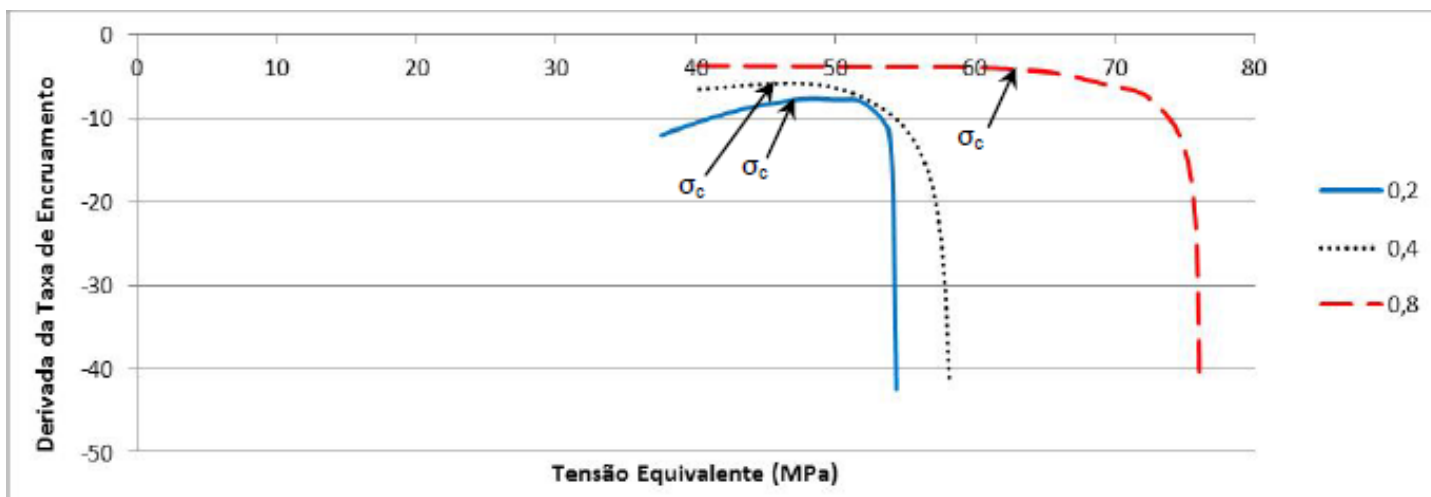

Figura 6: Curvas da derivada da Taxa de encruamento $(\mathrm{d} \theta / \mathrm{d} \sigma)$ versus a tensão equivalente $(\sigma)$ para um aço API SL X80 a diferentes taxas de deformação e temperatura de $1.150^{\circ} \mathrm{C}$.

* Contribuição técnica ao 69 Congresso Anual da ABM - Internacional e ao 14ํㅡㄹ ENET - Encontro Nacional de Estudantes de Engenharia Metalúrgica, de Materiais e de Minas, 21 a 25 de julho de 2014, São Paulo, SP, Brasil. 


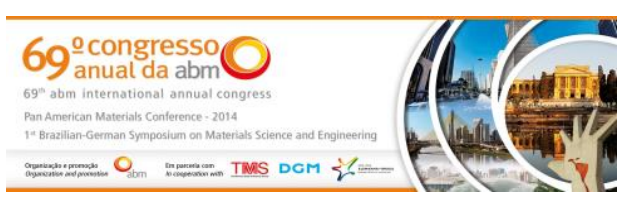

A partir das figuras 3,5 e 6 , organizou-se os parâmetros termomecânicos encontrados, como tensão de pico $\left(\sigma_{p}\right)$, tensão crítica $\left(\varepsilon_{c}\right)$, deformação de pico $\left(\varepsilon_{p}\right)$, deformação critica $\left(\varepsilon_{c}\right)$ e tensão no estado estacionário $\left(\sigma_{s s}\right)$, como mostra a Tabela 2.

Tabela 2 - Composição química do API $5 \mathrm{~L}$ X80 (com alto $\mathrm{Nb}$ )

\begin{tabular}{ccccccc}
$\dot{\varepsilon}\left(\mathbf{s}^{-1}\right)$ & $(\mathrm{MPa})$ & $(\mathrm{MPa})$ & $\boldsymbol{\varepsilon}_{\mathrm{p}}$ & $\boldsymbol{\varepsilon}_{\mathrm{c}}$ & $\boldsymbol{\varepsilon}_{\mathrm{c}} / \varepsilon_{\mathrm{p}}$ & $\begin{array}{c}\mathbf{\sigma}_{\mathbf{s s}} \\
(\mathrm{MPa})\end{array}$ \\
\hline $\mathbf{0 , 2}$ & 57,4 & 48,5 & 0,36 & 0,17 & 0,47 & 50,5 \\
$\mathbf{0 , 4}$ & 58,8 & 46,7 & 0,46 & 0,23 & 0,50 & 53,1 \\
$\mathbf{0 , 8}$ & 76,2 & 64,1 & 0,62 & 0,35 & 0,57 & 68,3
\end{tabular}

$\mathrm{Na}$ Figuras 7 pode-se verificar um aumento da tensão de pico a medida que a temperatura decresce, e que a mesma mostra similaridade com outros estudos realizados através de ensaio de torção a quente $[5,2,8]$. Este é um comportamento esperado visto que a densidade e distribuição de discordâncias, assim como a energia armazenada na deformação são fatores que dependem diretamente da temperatura durante a deformação do metal [8-10]. Com o decréscimo da temperatura a mobilidade das discordâncias diminui, causando o aumento da tensão e um deslocamento das curvas para a esquerda mostrando que existe um encruamento mais fácil do material.

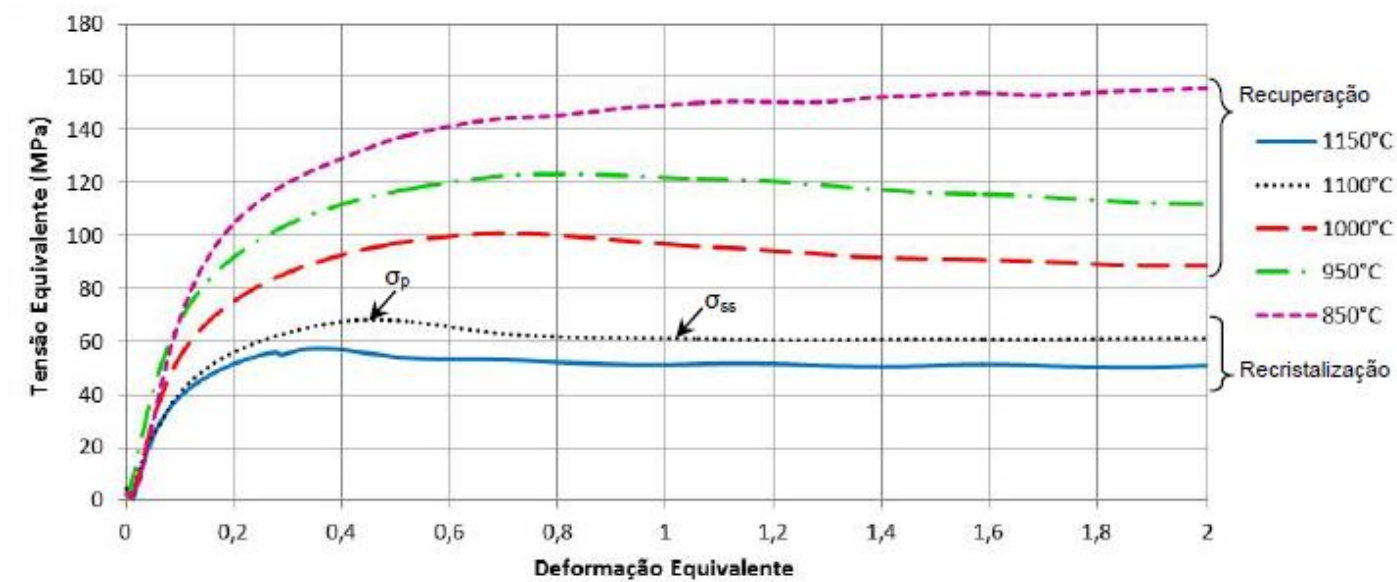

Figura 7: Curvas Tensão equivalente versus deformação equivalente, obtidas por ensaios de torção a quente para um aço API SL X80 a diferentes temperaturas e taxa de deformação constante de $0,2 \mathrm{~s}^{-1}$.

Observe-se que para as temperaturas de $1.000^{\circ} \mathrm{C}, 950^{\circ} \mathrm{C}$ e $850^{\circ} \mathrm{C}$, o comportamento das curvas é de material que se recupera dinamicamente, ou seja, ocorre maior encruamento no inicio da deformação até atingir o pico de tensão. Temperaturas mais baixas há a ocorrência de encruamento, uma vez que ocorre a recuperação do metal se faz mais lentamente.

Para as temperaturas de $1.150^{\circ} \mathrm{C}$ e $1.100^{\circ} \mathrm{C}$, observa-se que as curvas de escoamento plástico apresentam um comportamento típico de materiais que se recristalizam dinamicamente. Inicialmente há um aumento da tensão de escoamento plástico com a deformação até atingir um valor de pico, e em seguida ocorre uma diminuição da tensão até um estado estacionário.

A figura 8 mostra os ensaios realizados com taxa de deformação de $0,2 \mathrm{~s}^{-1}$ para temperaturas de deformação acima da $\mathrm{T}_{\mathrm{nr}}$.

* Contribuição técnica ao 69 Congresso Anual da ABM - Internacional e ao 14ํㅡㄹ ENET - Encontro Nacional de Estudantes de Engenharia Metalúrgica, de Materiais e de Minas, 21 a 25 de julho de 2014, São Paulo, SP, Brasil. 

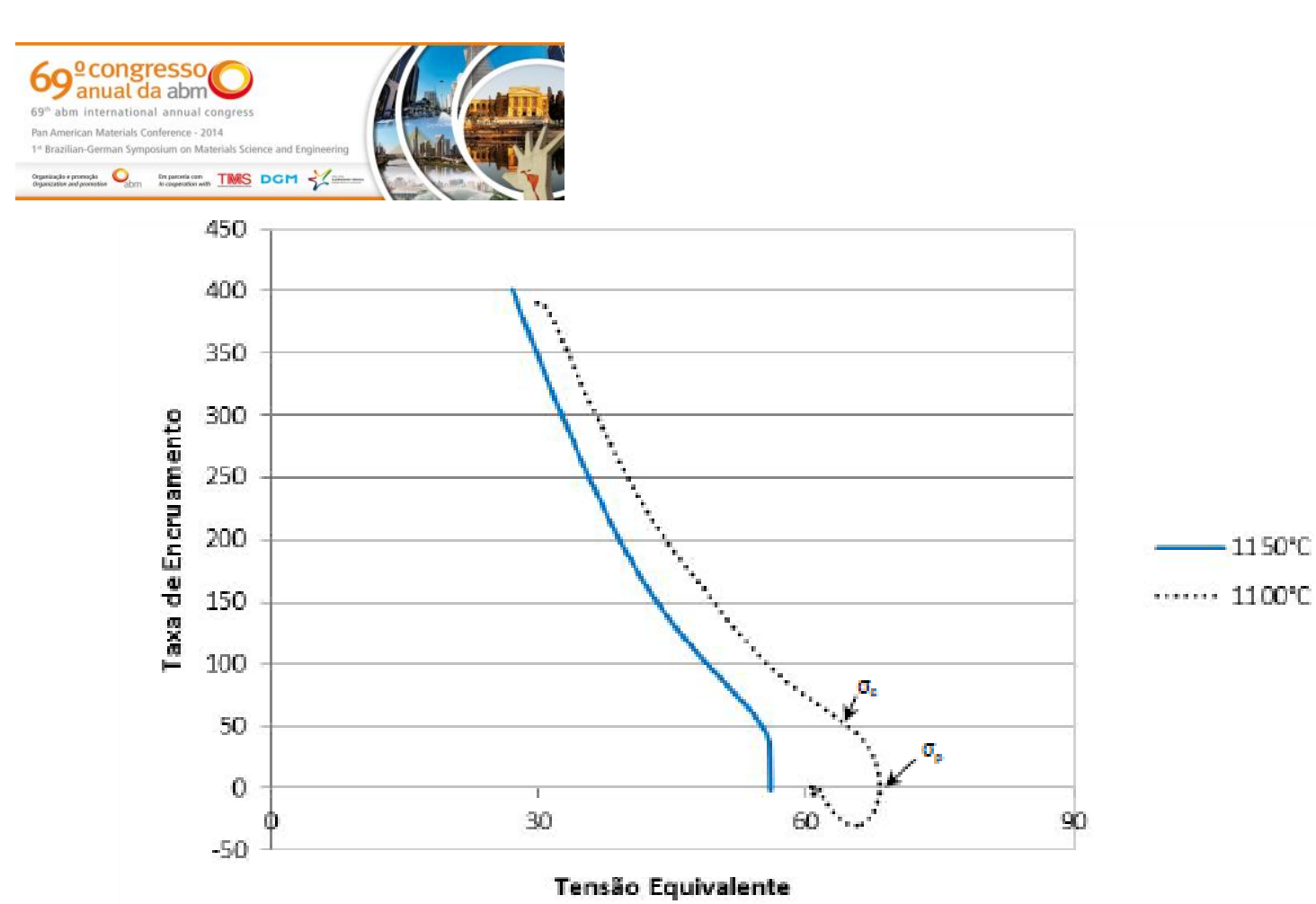

Figura 8: Variação da Taxa de encruamento $(\theta)$ versus a tensão equivalente para um aço API $5 \mathrm{~L}$ X80 a diferentes temperaturas a taxa de deformação constante de $0,2 \mathrm{~s}^{-1}$.

Observa-se uma mudança no comportamento das curvas, devido ao acúmulo de discordâncias e surgimento de subgrãos [7]. A taxa de encruamento diminui com 0 aumento da tensão inicialmente de forma linear e depois de forma parabólica.

$\mathrm{Na}$ curva da figura 8 pode-se observar um ponto de inflexão, que corresponde a tensão crítica $\left(\sigma_{c}\right)$ para inicio da recristalização dinâmica. Colocando-se o valor obtido na curva da figura 8 da tensão crítica $\left(\sigma_{c}\right)$ na curva de tensão versus deformação, encontra-se o valor da deformação crítica $\left(\varepsilon_{c}\right)$. A taxa de encruamento decresce até um valor de tensão máxima, que corresponde a tensão de pico $\left(\sigma_{p}\right)$, onde a taxa de encruamento $(\theta)$ é zero.

Os dados obtidos das figuras 7 e 8 foram organizados na Tabela 3.

Tabela 2 - Valores obtidos nos ensaios isotérmicos para um aço API $5 \mathrm{~L}$ X80 a diferentes temperaturas e taxa de deformação constante de $0,2 \mathrm{~s}^{-1}$

\begin{tabular}{ccccccc}
$\begin{array}{c}\text { Temperatura } \\
\left({ }^{\circ} \mathrm{C}\right)\end{array}$ & $\begin{array}{c}\sigma_{\mathrm{p}} \\
(\mathrm{MPa})\end{array}$ & $\begin{array}{c}\sigma_{\mathrm{e}} \\
(\mathrm{MPa})\end{array}$ & $\varepsilon_{\mathrm{p}}$ & $\varepsilon_{\mathrm{e}}$ & $\boldsymbol{\varepsilon}_{\mathrm{d}} / \varepsilon_{\mathrm{p}}$ & $\begin{array}{c}\sigma_{\mathrm{ss}} \\
(\mathbf{M P a})\end{array}$ \\
\hline $\mathbf{1 . 1 5 0}$ & 57,4 & $\mathbf{4 8 , 5}$ & 0,36 & 0,17 & 0,47 & 50,5 \\
$\mathbf{1 . 1 0 0}$ & 68,3 & 59,7 & 0,45 & 0,24 & 0,53 & 61,1 \\
$\mathbf{1 . 0 0 0}$ & 100,8 & - & 0,70 & - & - & 88,6 \\
$\mathbf{9 5 0}$ & 123,4 & - & 0,80 & - & - & 107,0 \\
$\mathbf{8 5 0}$ & 155,6 & - & 1,99 & - & - & 155,6 \\
\hline
\end{tabular}

Observa-se que os valores de tensão de pico e tensão critica aumentam com decréscimo de temperatura.

\section{CONCLUSÃo}

O aço API $5 \mathrm{~L}$ X80 ao ser submetido ao ensaio de torção a quente isotérmico em uma temperatura de $1.150{ }^{\circ} \mathrm{C}$ apresentou um comportamento típico de materiais que se recristalizam dinamicamente

\footnotetext{
* Contribuição técnica ao 69 Congresso Anual da ABM - Internacional e ao $14^{\circ}$ ENEMET - Encontro Nacional de Estudantes de Engenharia Metalúrgica, de Materiais e de Minas, 21 a 25 de julho de 2014, São Paulo, SP, Brasil.
} 
Ao submeter o material a um ensaio com diferentes temperaturas e taxa de deformação constante, observa-se que ocorre o aumento da tensão de pico a medida que a temperatura decresce.

Em temperaturas de $1.000^{\circ} \mathrm{C}, 950^{\circ} \mathrm{C}$ e $850^{\circ} \mathrm{C}$, o comportamento das curvas é de material que se recupera dinamicamente.

A $1.150^{\circ} \mathrm{C}$, o comportamento das curvas é de material que se recristaliza dinamicamente.

\section{REFERÊNCIAS}

1 Barbosa R. Simulação de processos industriais a partir do ensaio de torção a quente, Minas Gerais, Brasil, Escola de Engenharia da Universidade Federal de Minas Gerais.

2 Regone W. Simulação da laminação a quente de um aço livre de intersticiais (if) através de ensaios de torção, São Paulo, FAPESP, 2001.

3 Ferraz H. Aço na construção civil, São Paulo, Escola de Engenharia de São Carlos da Universidade de São Paulo, 2005.

4 Cho SH, Yoo CY. Hot rolling simulations of austenitic stainless steel. Journal of Materials Science, v. 36, p. 4267-4272, Abril 2001.

5 Geittens AR. Análse do comportamento mecânico de um aço carbono processado na região intercrítica. São Paulo: UFSCAR, 2001.

6 Balancin O. Tese de Doutorado, p. 25, 1981.

7 Tasca MRB. Análise do comportamento mecânico do aço SAE 1518 através de ensaior de torção a quente. Dissertação (Mestrado em Engenharia Matelúrgica e Materiais). Instituto Federal do Espirito Santo (IFES). Vitória, 2013.

8 Barcelos MV. Ensaios de torção a quente em aço estrutural. Vitória: IFES, 2011.

9 Padilha AF, Siciliano Jr.F. Encruamento, recristalização, crescimento de grão e textura. 3‥ ed. São Paulo: ABM, 2005.

10 Callister Jr. W.D. Ciência e engenharia de materiais: Uma introdução. 7º. ed. Rio de Janeiro: LTC, 2008.

* Contribuição técnica ao 69 Congresso Anual da ABM - Internacional e ao $14^{\circ}$ ENEMET - Encontro Nacional de Estudantes de Engenharia Metalúrgica, de Materiais e de Minas, 21 a 25 de julho de 2014, São Paulo, SP, Brasil. 